\title{
Comparison between Approximate and Exact Analytical Heat Conduction Rates in Struts of Rectangular Profile
}

\author{
Antonio Campo \\ Department of Mechanical Engineering, The University of Vermont, Burlington, VT 55455, USA
}

Corresponding Author Email: campanto@yahoo.com

https://doi.org/10.18280/ijht.370124

Received: 11 December 2018

Accepted: 4 March 2019

\section{Keywords:}

strut of rectangular profile, twodimensional heat conduction, transverse Biot number, slenderness ratio, temperature distribution, heat transfer rate, exact infinite series, approximate one term series

\begin{abstract}
The present study revolves around the quantification of heat transfer rates in struts of rectangular profile using an exact and two approximate solutions, which are expressed in terms of two controlling parameters: the transversal Biot number $B i_{t}$ and the slenderness ratio $S$. The exact, analytical formulation of the heat conduction problem for the strut of rectangular profile deals with the two-dimensional heat conduction equation, namely Laplace equation subject to four boundary conditions of Dirichlet, Neumann and Robin type. The exact, analytical solution is expressed by infinite series. One approximate solution uses the truncated one terms series. The approximate, analytical formulation relies on the standard quasi one-dimensional heat conduction equation subject to two boundary conditions of Dirichlet and Neumann type. The regions of validity of the two approximate heat transfer rates are delineated for engineering usage.
\end{abstract}

\section{INTRODUCTION}

Struts (or extended straight fins of rectangular profile) are extensively used in a multitude of heat exchange devices for the purpose of increasing the heat transfer rate between two opposed planar walls that are heated and an incoming fluid flowing at a low temperature between the planar walls [1].

From heat conduction theory, a strut transfers heat by longitudinal conduction within its defining boundaries, whereas the two exposed surfaces transfer heat by transverse convection to the fluid in which it is immersed. The heat conduction analysis in struts has been traditionally carried out through fin theory using the quasi one-dimensional heat conduction equation [1]. In general, the beneficial features of struts revolve around low mean convection coefficients, as is often the case for natural convection with air and gases or even in situations of forced convection with liquids moving at low velocities.

The formal mathematical formulation of a strut (extended straight fin) with rectangular profile must begin with the twodimensional heat conduction equation along with the proper boundary conductions [2]. The heat conduction problem has two figures-of-merit: one is the thickness $t$ ) and the other is the slenderness ratio $S=\frac{L}{t}$.

The dimensionality of straight fins of rectangular profile has been investigated by Lau and Tan [3], who performed a comparative numerical study of the heat transfer rates utilizing the formal two-dimensional heat conduction equation and the simplified quasi one-dimensional heat conduction equation. The authors used a combination of $B i_{t}=\frac{\bar{h} t}{k}$ (based on the half-thickness $t$ ) ranging between 0.01 and 10 and $S=\frac{L}{t}$ taking values from 1 up to 100 (resembling an infinite straight fin). In the publication, the relative errors between the two-dimensional and quasi one- dimensional heat transfer rates were reported in graphical form. Setting a relative error at $\varepsilon=5 \%$, it was found that the simplified quasi one-dimensional model rests on two components: 1) the transverse Biot number $B i_{t} \leq 0.5$ and 2) the slenderness ratio $S$ must be of order of 1 or greater. Subsequently, the relative error ascends to $\varepsilon=10 \%$ when $B i_{t}$ $=1$, irrespective of the value of $S$. Overall, the pattern displayed by the relative errors shows that $\varepsilon$ raises gradually with increments in $B i_{t}$, whereas $\varepsilon$ remains invariable to enlargements in $S$.

The primary objective of the present study is to utilize the physics-based two-dimensional heat conduction equation. The secondary objective is to develop a systematic procedure to establish a convenient criterion to assess the validity of the approximate, one-term series solution derived from the exact, infinite series solution of the two-dimensional heat conduction equation. The suited criterion shall be able to articulate the geometric, hydrodynamic and thermal quantities affecting the strut of rectangular profile. At the end, the transverse Biot number $B i_{t}$ united with the slenderness ratio $S$ establish the definitive borderline between the two-dimensional and the quasi one-dimensional heat transfer rates, along with the intrinsic relative errors for suitable pairs of $B i_{t}$ and $S$.

A literature review brings forth publications that gravitate around the exact two-dimensional heat conduction formulation for the straight fin of rectangular profile. Hence, the relevant publications are those by Sparrow and Hennecke [4], Look [5], Huang and Shah [6], Juca and Prata [7] and Singh et al. [8], which surprisingly did not touch upon the expedient approximate heat conduction formulations.

\section{FORMAL MATHEMATICAL MODEL}

A strut is an extended straight fin of rectangular profile that 
connects two opposed planar walls maintained at the same temperature $T_{b}$. In Figure 1, the proper dimensions of the strut are half-length $L$ in the $x$-direction, thickness $\delta=2 t$ in the $y$ direction and depth $w$ in the $z$-direction, which is very large in comparison with both $L$ and $\delta$. A viscous fluid at a low temperature $T_{f}$ flows perpendicular across the upper and lower parts of the strut. The thermal conductivity of the strut material $k$ is assumed to be constant or nearly invariant with temperature. For convenience, the variable convection coefficient $h$ over the strut surface is taken as the mean convection coefficient $\bar{h}$.

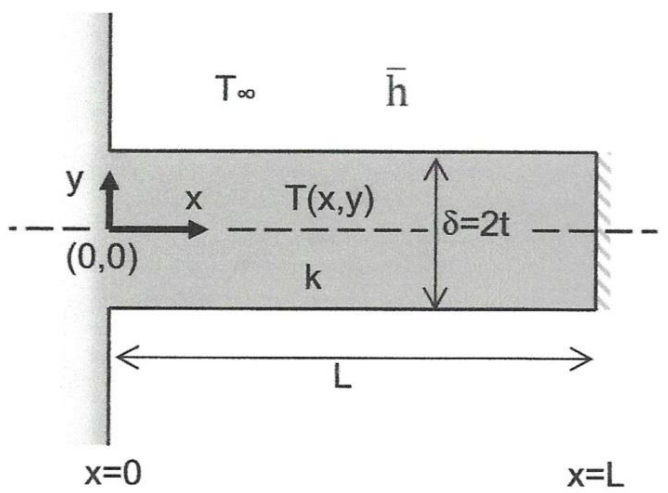

Figure 1. Sketch of the left part in a strut of rectangular profile Accordingly, the formal mathematical model for hte heat conduction problem implicates the two-dimensional heat conduction equation, i.e., Laplace's equation

$\frac{\partial^{2} \theta}{\partial x^{2}}+\frac{\partial^{2} \theta}{\partial y^{2}}=0$

where $\theta=T-T_{f}$ stands for the temperature excess.

The applicable boundary conditions for the upper left part of the strut are:

i) Prescribed temperature excess at the base:

$\theta=\theta_{b}, \quad x=0$, all $y$

ii) Thermal symmetry at the vertical mid-plane:

$\frac{\partial \theta}{\partial x}=0, \quad x=L$, all $y$

iii) Thermal symmetry at the horizontal mid-plane:

$\frac{\partial \theta}{\partial y}=0, \quad y=0$, all $x$

iv) Heat convection along the upper surface:

$-k \frac{\partial \theta}{\partial y}=\bar{h} \theta, \quad y=t$, all $x$

Collectively, the mixed boundary conditions in Eqns. (2a)(2b) are of Dirichlet, null Neumann and Robin type.

With regards to the experimental determination of the mean convection coefficient $\bar{h}$ showing up in eq. (2d), Moffat [9] has stated that the uncertainties in $\bar{h}$ have relative errors that usually range between $\pm 10 \%$ and $\pm 25 \%$. The different sources of relative errors are: (1) Instrumentation errors, (2)
Calibration errors and (3) Errors inherent to the regression equation in the analysis of the experimental data.

\section{EXACT ANALYTICAL SOLUTION}

The method of separation of variables engages the product of two functions

$$
\theta(x, y)=X(x) Y(y)
$$

and transforms the elliptic partial differential Eq. (1) into a system of two ordinary differential equations of second order [2]. One ordinary differential equation of second order associated with the $y$-direction is

$\frac{d^{2} Y}{d y^{2}}+\lambda^{2} Y=0$

subject to two homogeneous boundary conditions

$\frac{d Y(0)}{d y}=0$

$-k \frac{\partial Y(t)}{\partial y}=\bar{h} Y(t)$

coming from Eqns. (2c) and (2d). Correspondingly, the set of Eqns. (4), (5a) and (5b) defines an eigenvalue problem in the $y$-direction. Another ordinary differential equation of second order connected to the $x$-direction is

$\frac{d^{2} X}{d x^{2}}-\lambda^{2} X=0$

subject to a homogeneous boundary condition

$\frac{d X(L)}{d x}=0$

and a non-homogeneous boundary condition

$X(0)=\theta_{b}$

coming from Eqns. (2a) and (2b).

Conceptually, the set of Eqns. (6) and (7a) and (7b) defines a boundary value problem (BVP) in the $x$-direction.

On one hand, the general solution of eq. (6) is

$Y_{n}(y)=C_{n} \cos \lambda_{n} y$

where $C_{n}$ is an arbitrary constant and $\lambda_{n}$ are the roots of the transcendental equation

$k \lambda_{n}\left(\sin \lambda_{n} t\right)=\bar{h}\left(\cos \lambda_{n} t\right)$

On the other hand, the general solution of Eq. (4) is expressed in terms of exponential functions

$X_{n}(x)=B_{n}\left[e^{\lambda_{n} x}+e^{(2 L-x) \lambda_{n}}\right]$ 
where $B_{n}$ is an arbitrary constant.

In compliance with the trio of Eqns. (3), (8) and (10), the product solution $\theta(x, y)$ is next expressible by the infinite series

$\theta(x, y)=\sum_{n=1}^{\infty} a_{n}\left[e^{\lambda_{n} x}+e^{(2 L-x) \lambda_{n}}\right] \cos \lambda_{n} y$

where the new coefficients are

$a_{n}=B_{n} C_{n}$

Substituting the boundary condition for the base temperature excess $\theta_{b}$ stated in Eq. (7b) into Eq. (11), delivers the infinite series

$\theta_{b}=\sum_{n=1}^{\infty} a_{n}\left(1+e^{2 L \lambda_{n}}\right) \cos \lambda_{n} y$

The mathematical meaning of this equation signifies that the function $f(y)=\theta_{b}$ needs to be represented in terms of a Fourier series, where the unknown coefficients $a_{n}\left(1+e^{2 L \lambda_{n}}\right)$ are Fourier series coefficients. These Fourier series coefficients $a_{n}\left(1+e^{2 L \lambda_{n}}\right)$ are obtained from the expansion of the cosine Fourier series in Eq. (13) in a series of orthogonal functions. On account of this, the appropriate formula found in Arpaci [2] is

$a_{n}\left(1+e^{2 L \lambda_{n}}\right)=\frac{\int_{0}^{1} \theta_{b} \cos \lambda_{n} y d y}{\int_{0}^{1} \cos ^{2} \lambda_{n} y d y}$

Performing the integrations while skipping the algebra, the coefficients $a_{n}\left(1+e^{2 L \lambda_{n}}\right)$ are given at the end by the equation

$a_{n}\left(1+e^{2 L \lambda_{n}}\right)=2 \theta_{b}\left(\frac{\sin \lambda_{n} t}{\lambda_{n} t+\sin \lambda_{n} t \cos \lambda_{n} t}\right)$

Introducing Eq. (15) into Eq. (12), delivers the infinite series

$\theta(x, y)=2 \theta_{b} \sum_{n=1}^{\infty} \frac{1}{\left(1+e^{2 L \lambda_{n}}\right)}\left(\frac{\sin \lambda_{n} t}{\lambda_{n} t+\sin \lambda_{n} t \cos \lambda_{n} t}\right)\left[e^{\lambda_{n} x}+\right.$ $\left.e^{(2 L-x) \lambda_{n}}\right] \cos \lambda_{n} y$

which is the exact, analytical solution of Eq. (1) subject to Eqns. (2a)-(2d).

Returning to Eq. (9), the simultaneous effect of the mean convection coefficient $\bar{h}$ and the thermal conductivity $k$ on the roots $\lambda_{n}$ are adequately encapsulated into a thermo-geometric parameter. That is, knowing that the internal conductive resistance is $R_{K}=\frac{t}{k A}$ and the external convective resistance is $R_{C}=\frac{1}{\bar{h} A}$, their ratio sets up the transverse Biot number $B i_{t}$ :

$\frac{R_{K}}{R_{C}}=\frac{t / k A}{1 / \bar{h} A}=\frac{\bar{h} t}{k}=B i_{t}$

where the half-thickness of the strut $t$ acts as the characteristic length.

With the previous background, and resorting to the eigenvalues $\mu_{n}=\lambda_{n} t$ for compactness, Eq. (9) is reformulated into the dimensionless transcendental equation $\mu_{n} \tan \mu_{n}=B i_{t}$

For a given $B i_{t}$ inside the range $0<B i_{t}<\infty$, there is an infinite number of positive eigenvalues $\mu_{n}$ that satisfy eq. (18). The first six eigenvalues $\mu_{n}(n=1, \ldots, 6)$ are available in the heat conduction textbooks by Schneider [10], Luikov [11] and Özişik [12]. Advantageously, the entire set of eigenvalues $\mu_{n}=f\left(B i_{t}\right)$ can be computed nowadays with explicit equations developed by Milkhailov and Vulchanov [13] and Haji-Sheik and Beck [14].

In view of the foregoing, Eq. (16) can be rewritten in terms of the eigenvalues $\mu_{n}$ as follows

$\theta(x, y)=$
$2 \theta_{b} \sum_{n=1}^{\infty} \frac{\left[e^{\left.\mu_{n} \frac{x}{t}+e^{\frac{1}{t}(2 L-x) \mu_{n}}\right]}\right.}{\left(1+e^{2 \frac{L}{t} \mu_{n}}\right)}\left(\frac{\sin \mu_{n}}{\mu_{n}+\sin \mu_{n} \cos \mu_{n}}\right) \cos \mu_{n} \frac{y}{t}$

Actually, Eq. (19) embodies the exact, analytical twodimensional temperature distribution in the strut of rectangular profile under study. Certainly, this equation is useful in gaining physical insight into the heat conduction features in the strut.

The exact heat transfer rate from the strut of rectangular profile to the nearby fluid is determined from Fourier's law applied at the left base of the strut $x=0$ :

$Q_{2-D}=-2 k w \int_{0}^{t} \frac{\partial \theta(0, y)}{\partial x} d y$

Next, after introducing the temperature distribution $\theta(x, y)$ from Eq. (19), $Q$ is represented by the infinite series

$Q_{2-D}=4 k w \theta_{b} \sum_{n=1}^{\infty}\left(\frac{\sin ^{2} \mu_{n}}{\mu_{n}+\sin \mu_{n} \cos \mu_{n}}\right) \tanh S \mu_{n}$

where $S=\frac{L}{t}$ stands for the fin slenderness ratio.

In the two Eqns. (19) and (21) for the respective $\theta(x, y)$ and $Q_{2-D}$, the ensuing eigenvalues $\mu_{n}=f\left(B i_{t}\right)$ are determined from Eq. (18).

\section{APPROXIMATE ANALYTICAL SOLUTION}

Focusing on the exact infinite series in the pair of Eqns. (19) and (21), a particular case of importance deals with the approximate one-term series, which might be sufficient for engineering calculations.

From Sturm-Liouville theory [15], the set of eigenvalues $\mu_{n}$ in Eq. (18) follow the sequence of inequalities

$\mu_{1}<\mu_{2}<\ldots<\mu_{n-1}<\mu_{n}$

From here, it is recognized that the contribution of the first eigenvalue $\mu_{1}$ in the component $\frac{1}{1+e^{2 L \mu_{n}}}$ of the first term in Eqns. (19) becomes dominant. This issue signifies that the successive eigenvalues $\mu_{n}, n \geq 2$ in Eq. (19) can be taken as negligible for practical purposes. Consequently, the exact, infinite series in Eq. (19) can be approximated by the truncated one-term series, 
$\theta_{1}(x, y)=2 \theta_{b} \frac{\left[e^{\mu_{1} \frac{x}{t}}+e^{\frac{1}{t}(2 L-x) \mu_{1}}\right]}{\left(1+e^{2 \frac{L}{t} \mu_{n}}\right)}\left(\frac{\sin \mu_{1}}{\mu_{1}+\sin \mu_{1} \cos \mu_{1}}\right) \cos \mu_{1} \frac{y}{t}(23)$

related to the set of transverse Biot numbers $B i_{t}$ in the range 0 $<B i_{t}<\infty$.

Analogously, from Eq. (21) the equivalent one-term series that quantifies the heat transfer rate simplifies to

$Q_{2-D, 1}=4 k w \theta_{b}\left(\frac{\sin ^{2} \mu_{1}}{\mu_{1}+\sin \mu_{1} \cos \mu_{1}}\right) \tanh S \mu_{1}$

In the pair of Eqns. (23) and (24), $\mu_{1}$ represents the first eigenvalue from the transcendental equation

$\mu_{1} \tan \mu_{1}=B i_{t}$

coming from Eq. (18).

\subsection{Regression analysis of the $\mu_{1}=f\left(B i_{t}\right)$ data}

The first eigenvalue $\mu_{1}$ depends on $B i_{t}$ spanning from a very low $B i_{t}=0.01$ to an extremely high $B i_{t}=100$ (resembling prescribed surface temperature). A table listing $\mu_{1}$ in terms of $B i_{t}$ is available in the textbooks on heat transfer by Luikov [11] and Özişik [12].

It is obvious that reading numbers from tables is inaccurate, inconvenient and time consuming for engineering calculations. Even more, for those $B i_{t}$ values not listed in the table, the adequate estimation of $\mu_{1}$ forcibly requires quadratic interpolation.

In light of the foregoing, the tabulated $\mu_{1}^{2}$ vs $B i_{t}$ data reported in Luikov [11] and Özişik [12] has been treated with regression analysis. The outcome of the calculations delivers the two-part correlation equation:

$\mu_{1}^{2}=B i_{t} \quad$ for $0 \leq B i_{t} \leq 0.1$

$\frac{1}{\mu_{1}^{2}}=0.40+\frac{0.92}{B i_{t}}$ for $0.1 \leq B i_{t} \leq 100$

covering the entire $B i_{t}$ spectrum $0<B i_{t}<100$.

\section{APPROXIMATE MATHEMATICAL MODEL}

The approximate mathematical model begins with the mean temperature excess:

$$
\bar{\theta}(x)=\frac{1}{t} \int_{0}^{t} \theta(x, y) d y
$$

where $\theta(x, y)$ is the two-dimensional temperature excess in Eq. (19).

A thermodynamic energy balance in a control volume of thickness $\Delta x$ placed in the strut (extended straight fin) of rectangular profile provides the quasi one-dimensional heat conduction equation

$$
\frac{d^{2} \bar{\theta}}{d x^{2}}-m^{2} \bar{\theta}=0
$$

where $m^{2}=\frac{\bar{h}}{k t}$ denotes the thermo-geometric parameter with units $1 / \mathrm{m}^{2}$ [16]. The boundary conditions for Eq. (28) are Eqns. (2a) and (2b) replacing $\theta$ with $\bar{\theta}$

$$
\begin{gathered}
\bar{\theta}=\theta_{b}, \quad x=0 \\
\frac{d \bar{\theta}}{d x}=0, \quad x=L
\end{gathered}
$$

These boundary conditions are of Dirichlet and null Neumann type.

The general solution of Eq. (28) subject to Eqns. (29a) and (29b) gives the particular solution, which is expressed compactly as

$$
\frac{\bar{\theta}(x)}{\theta_{b}}=\frac{\cosh [m(L-x)]}{\cosh m L}
$$

This equation entails to the quasi one-dimensional mean temperature distribution in the strut of rectangular profile.

The heat transfer rate is calculated with Fourier's law applied at the base $x=0$ of the left part,

$Q_{\text {quasi } 1-D}=-k(2 t w) \frac{d \bar{\theta}(0)}{d x}$

where the cross-sectional area is $A_{c}=2 t w$. After introducing Eq. (30) into Eq. (31), the final expression for the heat transfer rate is

$Q_{\text {quasi } 1-D}=2 k w \theta_{b} \sqrt{B i_{t}} \tanh \left(S \sqrt{B i_{t}}\right)$

Herein, the thermo-geometric parameter $m$ and the transverse Biot number Bit are related by the equality $m^{2}=$ $\frac{B i_{t}}{t^{2}}$.

\section{PRESENTATION AND DISCUSSION OF RESULTS}

The suitability of the heat transfer analysis of struts of uniform profile must rely on the accurate calculation of the heat transfer rate $Q$. Therefore, the exact, analytical heat transfer rate given by the infinite series in Eq. (21) was evaluated with a symbolic computer code containing automatic convergence control between consecutive terms. A total of 212 terms were retained in Eq. (21) for combinations of the transverse Biot number $B i_{t}=0.01,0.1,05$ and 1 and slenderness ratios $S=1,5$, 10 and 100 .

First, the relative error $\varepsilon_{a}$ between the approximate $Q_{2-D, 1 \text { term }}$ in Eq. (24) and the exact $Q_{2-D}$ in Eq. (21) is defined by

$\varepsilon_{a}=\frac{Q_{2-D}-Q_{2-D, 1}}{Q_{2-D}}$

Second, the relative error $\varepsilon_{b}$ between the approximate $Q_{\text {quasi } 1-D}$ in Eq. (32) and the exact $Q_{2-D}$ in Eq. (21) is defined_by 
$\varepsilon_{b}=\frac{Q_{2-D}-Q_{\text {quasi } 1-D}}{Q_{2-D}}$

In general, it is observable in Table 1 that for all combinations of the transverse Biot numbers Bit $=0.01,0.1,0.5$ and 1 with the slenderness ratios $S=1,5$ and 10 , the relative errors $\varepsilon$ a for the one-term series are always less than the relative errors $\varepsilon b$ of the quasi one-dimensional solution. In particular, for combinations of Bit=0.5 and 1 along with all $S=1,5$ and 10 , the relative errors $\varepsilon b$ of the quasi one-dimensional model are very large, whereas the relative errors $\varepsilon a$ for the one-term series are acceptable. On one hand, for $B i_{t}=1$ with one $S=1$ the relative error of the one-term series is $\varepsilon_{a}=-8.43 \%$, the relative error of the two-term series is $-1.86 \%$ and the relative error of the three-term series is $-0.93 \%$. On the other hand, for $B i_{t}=1$ united to two $S=5$ and 10 the relative error of the one-term series is $\varepsilon_{a}=-6.03 \%$, the relative error of the two-term series is $1.33 \%$ and the relative error of the three-term series is $-0.67 \%$. As an additional observation, it should be pointed out that the relative error $\varepsilon_{b}=-10.8 \%$ associated with $B i_{t}=1$ together with $S=5$ and 10 provided by the approximate quasi one-dimensional model is consistent with the consonant relative error around $-10 \%$ found in the publication by Lau and Tan [3]. The approximate quasi one-dimensional solution for the straight fin of rectangular profile works well for struts of rectangular profile characterized by $B i_{t}=0.01$ and 0.1 coupled with $S=1,5$ and 10 ; all cases manifesting relative errors of less than $\varepsilon_{b}=-2 \%$. Firstly, increasing $B i_{t}$ to 0.5 articulated with $S=1$, the relative error climbs to $\varepsilon_{b}=-4.13 \%$, i.e., less than the acceptable relative error $-5 \%$ in engineering applications. Therefore, tolerating a relative error of $\varepsilon_{b}=-5 \%$ common in engineering calculations, and utilizing quadratic interpolation, $B i_{t}$ turns out to be 0.31 when $S=5$, and $B i_{t}$ turns out to be 0.43 when $S=10$. Secondly, increasing $B i_{t}$ to 1 for all $S=1,5$ and 10, the approximate quasi one-dimensional solution for the straight fin of rectangular profile contains large relative errors that are equal or exceed $\varepsilon_{b}=-10.80 \%$ evidencing that it does not work.

Table 1. Relative errors $\varepsilon$ of the approximate heat transfer rates $Q_{2-D, 1 \text { term }}$ and $Q_{\text {quasi I-D }}$ with respect to the exact heat transfer rate $Q_{2-D}$ for combinations of the transverse Biot number $B i_{t}$ and the slenderness ratio $S$

\begin{tabular}{|c|c|c|c|}
\hline$B i_{t}=\frac{\bar{h} t}{k}$ & $S=\frac{L}{t}$ & $\begin{array}{c}\text { Approx. } \\
\text { 2-D } \\
\text { model with one } \\
\text { term series } \\
\varepsilon_{a} \%\end{array}$ & $\begin{array}{c}\text { Approx. } \\
\text { quasi 1-D } \\
\text { model } \\
\varepsilon_{b} \%\end{array}$ \\
\hline 0.01 & 1 & -0.078 & -0.26 \\
\hline 0.01 & 5 & -0.017 & -0.29 \\
\hline 0.01 & 10 & -0.010 & -0.25 \\
\hline 0.1 & 1 & -0.39 & -0.25 \\
\hline 0.1 & 5 & -0.26 & -1.88 \\
\hline 0.1 & 10 & -0.24 & -1.48 \\
\hline 0.5 & 1 & -4.07 & -4.13 \\
\hline 0.5 & 5 & -2.07 & -7.41 \\
\hline 0.5 & 10 & -2.04 & -6.29 \\
\hline 1 & 1 & -8.43 & -18 \\
\hline 1 & 5 & -6.03 & -11 \\
\hline 1 & 10 & -6.03 & -11 \\
\hline & & & \\
\hline
\end{tabular}

The truncated approximate one term series for a relative large $B i_{t}=1$ united to $S=1,5$ and 10 exhibits relative errors that slightly exceed $-5 \%$. Therefore, if a relative error is fixed at the traditional $\varepsilon_{b=}-5 \%$, then the corresponding $B i_{t}$ values computed with quadratic interpolation are: $0.60 S=1$, 0.92 for $S=5$ and 10 .

\section{CONCLUSIONS}

The main conclusions that may be drawn from the present study on the determination of heat transfer rates in twodimensional struts of rectangular profile are listed next:

1) An exact, analytical solution of the two-dimensional heat conduction equation subject to the proper boundary conditions provides the exact benchmark heat transfer rates represented by an infinite series in terms of two controlling parameters: the transversal Biot number $B i_{t}$ and the slenderness ratio $S$.

2) The approximate solution of the quasi one-dimensional heat conduction equation for the straight fin of rectangular profile works well for two-dimensional struts of rectangular profile when the transversal Biot numbers are small of the order of $B i_{t}=0.01$ and 0.1 in conjunction with the slenderness ratios $S=1,5$ and 10; all cases manifest relative errors $\varepsilon$ of less than $2 \%$. For $B i_{t}>0.5$ regardless of $S$, the relative errors increase significantly.

3) Numerical evaluation of the exact infinite series solution of the two-dimensional heat conduction equation is not necessary. It was demonstrated in the present study that the approximate one term series solution display relative errors $\varepsilon$ that are less than $-5 \%$ whenever the transversal Biot number $B i_{t} \leq 0.60$ is combined with the slenderness ratio $S=1$. Additionally, the same relative error criterion of $-5 \%$ applies to situations when the transversal Biot number $B i_{t} \leq 0.92$ is combined with the slenderness ratios $S=5$ and 10 .

\section{ACKNOWLEDGEMENTS}

The author praises Dr. Antonio Acosta-Iborra for carrying out some calculations during the first phase of the study.

\section{REFERENCES}

[1] Kraus AD, Aziz A, Welty J. (2001). Extended surface heat transfer. John Wiley and Sons, Hoboken, NJ, USA.

[2] Arpaci V. (1966). Conduction Heat Transfer, AddisonWesley, Reading, MA, USA.

[3] Lau W, Tan CW. (1973). Errors in one-dimensional heat transfer analysis in straight and annular fins. Journal of Heat Transfer 95(4): 549-551. https://doi.org/10.1115/1.3450110

[4] Sparrow E, Hennecke DK. (1970). Temperature depression at the base of a fin. Journal of Heat Transfer 92(1): 204-206. https://doi.org/10.1115/1.3449636

[5] Look DJ. (1989). Two-dimensional fin with nonconstant root temperature. International Journal of Heat and Mass Transfer 32(5): 977-980. https://doi.org/10.1016/0017-9310(89)90247-0

[6] Huang L, Shah R. (1992). Assessment of calculation methods for efficiency of straight fins of rectangular profile. International Journal of Heat and Fluid Flow 13(3): 282-293. https://doi.org/10.1016/0142727X(92)90042-8

[7] Juca P, Prata AT. (1993). Two-dimensional fins attached to a thick wall: Effect of non-uniform root temperature. International Journal of Heat and Mass Transfer 36(1): 233-236. https://doi.org/10.1016/0017- 
9310(93)80084-8

[8] Singh IV, Sandeep K, Prakash R. (2003). Heat transfer analysis of two-dimensional fins using meshless element free Galerkin method. Numerical Heat Transfer, Part A: Applications 44(1): 73-84. https://doi.org/10.1080/713838174

[9] Moffat RJ. (1988). Describing the uncertainties in experimental results. Experimental Thermal and Fluid Science 1(1): 3-17. https://doi.org/10.1016/08941777(88)90043-X

[10] Schneider PJ. (1955). Conduction Heat Transfer, Addison-Wesley, Reading, MA, USA.

[11] Luikov AV. (1968). Analytical heat diffusion theory. Academic Press, London, England, UK.

[12] Özişik MN. (1993). Heat Conduction. 2nd edition, John Wiley and Sons, Hoboken, NJ, USA.

[13] Milkhailov MD, Vulchanov NL. (1983). Computational procedure for Sturm-Liouville problems. Journal of Computational Physics 50(3): 323-336. https://doi.org/10.1016/0021-9991(83)90101-8

[14] Haji-Sheikh A, Beck JB. (2000). An efficient method of computing eigenvalues in heat conduction. Numerical Heat Transfer, Part B: Fundamentals 38(2): 133-156. https://doi.org/10.1080/104077900750034643

[15] Hazewinkel M, (Editor). (2001). Sturm-Liouville Theory. In Encyclopedia of Mathematics, SpringerVerlag, Berlin, Germany.

[16] Mills AF. (1992). Heat Transfer. 2nd edition, CRC Press, Boca Raton, FL, USA.

\section{NOMENCLATURE}

$a_{n}$

A

$A_{c}$

$B i_{t}$
Fourier series coefficients in eq. (13) surface area of strut $\left(\mathrm{m}^{2}\right)$ cross-sectional area of strut, $2 t w\left(\mathrm{~m}^{2}\right)$ transverse Biot number, $\frac{\bar{h} t}{k}$ convection coefficient $\left(\mathrm{W} / \mathrm{m}^{2} \mathrm{~K}\right)$

mean convection coefficient $\left(\mathrm{W} / \mathrm{m}^{2} \mathrm{~K}\right)$

thermal conductivity $(\mathrm{W} / \mathrm{m} \mathrm{K})$

half-length of strut (m)

thermo-geometric parameter, $\left(1 / \mathrm{m}^{2}\right)$

heat transfer rate $(\mathrm{W})$

external convection resistance (K/W)

internal conduction resistance $(\mathrm{K} / \mathrm{W})$

slenderness ratio of strut, $\frac{L}{t}$

half-thickness of strut (m)

temperature $(\mathrm{K})$

base temperature $(\mathrm{K})$

fluid temperature $(\mathrm{K})$

transverse mean temperature $(\mathrm{K})$

depth of strut (m)

axial coordinate $(\mathrm{m})$

transversal coordinate $(\mathrm{m})$

\section{Greek symbols}

$\delta \quad$ thickness of strut, $2 t(\mathrm{~m})$

$\varepsilon \quad$ relative error

$\theta \quad$ temperature excess, $T-T_{f}(\mathrm{~K})$

$\bar{\theta} \quad$ transverse mean temperature excess, $\bar{T}-T_{f}(\mathrm{~K})$

$\lambda_{n} \quad$ roots of transcendental eq. (9)

$\mu_{n} \quad$ eigenvalues in eq. (18)

\section{Subscripts}

$\begin{array}{ll}\text { opt } & \text { optimal } \\ 1 & \text { one term }\end{array}$ 